\title{
QUALITY ASSURANCE IN ENVIRONMENTAL TECHNOLOGY VERIFICATION (ETV): ANALYSIS AND IMPACT ON THE EU ETV PILOT PROGRAMME PERFORMANCE
}

\author{
Michat MOLENDA \\ Silesian University of Technology \\ Izabela RATMAN-KŁOSIŃSKA \\ Institute for Ecology of Industrial Areas
}

\begin{abstract}
:
Many innovative environmental technologies never reach the market because they are new and cannot demonstrate a successful track record of previous applications. This fact is a serious obstacle on their way to the market. Lack of credible data on the performance of a technology causes mistrust of investors in innovations, especially from public sector, who seek effective solutions however without compromising the technical and financial risks associated with their implementation. Environmental technology verification (ETV) offers a credible, robust and transparent process that results in a third party confirmation of the claims made by the providers about the performance of the novel environmental technologies. Verifications of performance are supported by high quality, independent test data. In that way ETV as a tool helps establish vendor credibility and buyer confidence. Several countries across the world have implemented ETV in the form of national or regional programmes. ETV in the European Union was implemented as a voluntary scheme if a form of a pilot programme. The European Commission launched the Environmental Technology Pilot Programme of the European Union (EU ETV) in 2011. The paper describes the European model of ETV set up and put to operation under the Pilot Programme of Environmental Technologies Verification of the European Union. The goal, objectives, technological scope, involved entities are presented. An attempt has been made to summarise the results of the EU ETV scheme performance available for the period of 2012 when the programme has become fully operational until the first half of 2016. The study was aimed at analysing the overall organisation and efficiency of the EU ETV Pilot Programme. The study was based on the analysis of the documents the operation of the EU ETV system. For this purpose, a relevant statistical analysis of the data on the performance of the EU ETV system provided by the European Commission was carried out.
\end{abstract}

Key words: enviromental technology verification, quality assurance, environmental technologies, ecoinnovation

\section{INTRODUCTION}

There is an increasing environmental awareness of the international community the challenges posed to the environment due to human activity such as climate change, air pollution, increasing water scarcity, depleting natural resources or loss of biodiversity. It has been reflected in the concept of sustainability which has been constantly developed in theory and intensively implemented in practice in order to ensure economic growth without compromising the ability of future generations to meet their own needs. Achievement of sustainability is currently one of the most important considerations to be addressed by the international community. To make progress towards sustainability, efforts are essential that would ensure that the environmental, social and economic systems develop in a balanced and mutually supporting ways. Environmental technologies i.e. technologies which are more beneficial or cause less adverse environmental impact compared to relevant alternatives, play an increasing role in achieving the sustainability objective. At the same time they contribute positively to competitiveness and growth.

Despite an increasing demand of the market for innovations and the environmental awareness of technology buy- ers, breaking into the market with ecoinnovations is a significant problem, because contrary to established technologies innovations by definition cannot demonstrate a successful track-record of applications. Often, cutting edge environmental technologies encounter problems in demonstrating their environmental added value and proving benefits to users resulting from their implementation.

Without credible information potential buyers are unsure whether or not to trust the vendors' claims made about the performance of offered solutions. This significantly hampers both the marketability of new environmental technologies and the development of small and medium enterprises. In majority of cases vendors operate in highly competitive market conditions. In 1995, a system enabling verification of performance of environmental technologies was first established as a systemic tool in the United States of America followed by other countries such as Canada, South Korea, Japan or Philippines. The system was developed to overcome the barriers in the market uptake of new environmental technologies that support environmental policy objectives. Despite the differences among the ETV systems worldwide, all of them operate based on quality test data and transparent verification procedures per- 
formed by competent organisations of a recognised reputation. A common objective for all these systems is to produce a verification result that would provide confidence to the purchasers that a vendor's claim of performance for an environmental technology is valid, credible, supported by quality independent test data and based on solid scientific and engineering principles.

Recognising similar problems in the market penetration of ecoinnovative technologies and benefiting from the experiences of the ETV forerunners, the European Commission launched an EU ETV scheme in 2011 as a pilot action under the Ecoinnovation Action Plan.

The launch of the programme was preceded by a feasibility study carried out by the Commission's Joint Research Centre - Institute for Prospective Technological Studies [1]. Within these studies potential stakeholders were interviewed on the usefulness of the system. One of the main issues indicated by the respondents making ETV a useful market tool was that the system represented a guarantee for quality of information on a technology. Another consultation on ETV launched [2] by the European Commission together with the European Business Test Panel showed that only $11 \%$ of purchasers trusted vendors' claims; more than half $(57 \%)$ would ask for evidence backing-up the claims and $26 \%$ considered they had no way of checking the declared performance. At the same time, out of the total of 470 questioned participants, $69 \%$ considered there was a clear or important need to promote and organise a third party verification of technology performance. As the first objective for an ETV scheme $48 \%$ indicated "Help technology purchasers (public or private) to base their purchase decision on reliable information". This data clearly demonstrated that quality assurance is one of the issues of critical importance for establishing a successful ETV scheme in Europe and thus provide for the usefulness and reliability of the verification results.

The paper describes the European model of ETV set up and put to operation under the Pilot Programme of Environmental Technologies Verification of the European Union. The goal, objectives, technological scope, involved entities and the verification procedures are presented. An attempt has been made to summarise the results of the EU ETV scheme performance in the context of quality assurance available for the period of 2012 when the programme has become fully operational until the first half of 2016. The study was aimed at analysing the overall organisation of the EU ETV Pilot Programme, its goals, technological scope, involved entities and the verification procedures against the requirements of quality assurance. The study was based on the analysis of the documents regulating the operation of the EU ETV system, detailing its procedures and providing additional guidance on their implementation. These documents are specified further on in the section presenting the references. Moreover, a relevant statistical analysis of the data on the performance of the EU ETV system provided by the European Commission was carried out. It enabled a quantitative description of some aspects related to quality assurance in the system operation.

\section{GENESIS AND CONCEPT OF THE EU ETV SCHEME}

The concept of environmental technology verification is not new. It has been described in literature $[3,4,5,6,7,8]$ as well as deployed in a number of programmes and schemes operational worldwide. ETV schemes have been implemented for nearly two decades in North America (US and Canada) and for half a decade in East Asia (Japan, Korea, the Philippines). In Europe, several research and pilot projects have been funded by the EU FrameworkProgramme for Research and Technological Development, by the Nordic Innovation Council and by several Member States (Denmark, Germany, the Netherlands).

The European Commission launched the Pilot Programme of Environmental Technologies Verification of the European Union under the auspices of DG Environment on 15 December 2011, together with the adoption of the Ecoinnovation Action Plan (EcoAP). More specifically, the programme is one of the tools in under EcoAP's action 4 dedicated, among others, to mobilise support to SMEs in order to improve investment readiness, networking opportunities as well as market confidence in eco-innovation.

The EU ETV scheme is based on the experiences gathered from the American and Asian programmes as well as national and regional ETV programmes set up in Scandinavian countries. They were collected, analysed and transformed into the foundations of the EU ETV programme under four EU research projects carried out between 2004 and 2009, in the fields of water treatment, soil and groundwater remediation, air emissions abatement, clean production and environmental monitoring. These projects developed and tested protocols and procedures to implement ETV. Another project, AdvanceETV, began in 2009, was implemented to support the establishment of an EU-wide scheme as well as the international ETV harmonisation efforts.

The EU ETV Pilot Programme was established as a voluntary scheme with the participation of seven Member States: Belgium, the Czech Republic, Denmark, Finland, France, United Kingdom and Poland. Italy joined the group in 2014. One of the criteria to enter into the pilot programme was the readiness of the national accreditation bodies to perform accreditations of potential verification bodies for compliance to ISO/IEC 17020 for inspection bodies type A to perform verifications according to the General Verification Protocol of the EU ETV Pilot Programme.

Quite similar as in the case of other ETV systems, the EU ETV was established with the following objectives [9]:

- enable technology manufacturers, especially SMEs, to market their eco-technologies,

- give more credibility to developers of innovative technologies,

- enable technology purchasers (public or private) to benefit from innovation and select technologies fitting their needs,

- facilitate or accelerate the diffusion of ecoinnovative technologies on EU market and globally.

In terms of technological scope, although seven technology areas are mentioned in the General Verification Protocol, the EU ETV pilot phase has been limited to the following three technology areas:

1. Water treatment and monitoring (monitoring of water quality, treatment of drinking water and of wastewater).

2. Materials, waste and resources (separation and sorting of solid waste, recycling of materials, end-of-life products and chemicals, products from biomass).

3. Energy technologies (renewable sources of energy, energy from waste, energy efficiency technologies).

From the quality assurance viewpoint, the criteria for selection of these technology areas included aspects such as availability of specific verification protocols, tech- 
nical standards, scientific studies or research providing a satisfactory pool of knowledge for the implementation of the verification procedures. Other issues considered were related to the availability of a significant number of test bodies having the necessary capacity and quality standards to generate quality test data. From the policy viewpoint, the aspect of needs in terms of technological development or quality requirements, emerging from EU and international policy developments were considered.

Other criteria included such aspects as the existence or emergence of a significant number of innovative environmental technologies - candidates for verification under ETV, the demand of technology developers and users, in particular SMEs for such service.

The EU ETV pilot programme is open to market-ready solutions from the above mentioned technology areas which demonstrate a potential for innovation and are of benefit to the environment. That means that a candidate technology for an EU ETV verification shall meet the definition of an environmental technology i.e. provide an environmental added value defined as the reduction of the environmental pressure or a positive impact on the environment including but not limited to removal, prevention, reduction, mitigation of pollutants released to the environment, restoration of environmental damages or use of natural resources in a more efficient and sustainable manner. Moreover, the technology should demonstrate innovative features in terms of design, raw materials and energy involved, production process, use, recyclability or final disposal, when compared with relevant alternatives. The EU ETV is targeted in particular at these technologies which perform better or whose benefits cannot be proved through existing standards or certification schemes.

Additionally to the environmental and innovation criteria, a candidate technology shall demonstrate adequate level of market readiness. In the aspect of quality assurance only sufficient level of technology development can guarantee that the verified data will reflect its actual and true performance. This means that a candidate technology for verification shall either be already available on the market or be developed at least to a stage of a prototype which could be characterised as technology readiness level 7 (TRL 7) i.e. system prototype demonstration in operational environment.

The entrance criteria for determining the eligibility of a technology for ETV include also such issues as the potential to meet user needs and its performance in line with applicable legal requirements. These issues are also linked to the quality assurance aspects of EU ETV. The fact that a technology does not comply with the applicable legal regulations or absence of sufficient evidence in that respect, may be a reason for rejecting the technology from verification.

Similarly as in the case of other ETV schemes, the key feature distinguishing the EU ETV from certification or conformity assessment standards is the fact that it is not a pass -or-fail system based on a technology assessment against a set of predefined criteria or standards. The result of EU ETV is a validated declaration proposed by the vendor on the performance of a technology expressed by a set of parameters and their numerical values most relevant to demonstrate the benefits and the environmental added value of a technology. Moreover, unlike certification schemes, EU ETV allows for flexibility in specifying the performance parameters to maximize the usefulness of the verification results.
To achieve this, EU ETV procedure has been set up as a dynamic process allowing for a dialogue with the vendor in determining both the parameters to be verified and their values however without compromising the independence, transparency and credibility of the verification process.

\section{KEY DOCUMENTS OF THE EU ETV SCHEME}

As mentioned earlier, the need to support SMEs in marketing new environmental technologies and rising market confidence in ecoinnovations has been indicated in the COMMUNICATION FROM THE COMMISSION TO THE EUROPEAN PARLIAMENT, THE COUNCIL, THE EUROPEAN ECONOMIC AND SOCIAL COMMITTEE AND THE COMMITTEE OF THE REGIONS Innovation for a sustainable Future - The Eco -innovation Action Plan (Eco-AP). The EU ETV Pilot Programme is mentioned in this document as one of the initiatives to be launched by the European Commission. The principles and benefits of the EU ETV Pilot Programme have been further described in the Commission Staff Working Paper The Environmental Technology Verification (ETV) initiative Helping Eco-Innovations to reach the Market" attached to the Action Plan.

To support the development and implementation of the EU ETV Pilot Programme, a General Verification Protocol (GVP) has been elaborated. The GVP serves as the main technical reference for the implementation and coordination of the EU ETV process and procedures at the European level. The document specifies: i) an organisational and technical framework for the EU ETV, ii) procedures enabling the provision of independent and credible information on new environmental technologies in line with the objectives set up for the EU ETV Pilot Programme ii) the quality management and quality assurance of the scheme.

The use of the ISO standards allowed to set up the EU ETV scheme based on an existing quality assurance framework without the need to create dedicated conformity assessment requirements for the verification bodies. The reason for choosing compliance to the ISO/IEC 17020 standard as a requirement for the verification bodies was twofold: firstly the standard allows that conclusions are made based on professional judgment, secondly compliance to the requirements specified for inspection bodies type A guarantees the highest impartiality and credibility of the verifications. Moreover, in the meaning of ISO/IEC 17020 the verification process specified in the GVP should be understood as an inspection system for the verification bodies while the EU ETV Pilot Programme as an inspection scheme.

Another element of the quality assurance in the EU ETV is that the test system provided by the test bodies to generate test data used to back up the claimed performance must be compliant to the requirements of the ISO/IEC 17025 standard. Furthermore, the GVP specifies which measures need to be undertaken by the verification bodies towards the test bodies to assure quality at the absence of accreditation to ISO 17025 .

Establishment of the ETV Technical Working Groups and the development of the guidance documents in support of the GVP provide for an adequately harmonized performance of the verification bodies operating under the EU ETV in terms of implementing the verification procedures. Thus, from the viewpoint of the provisions defined for the EU ETV in the GVP, the guidance documents and the applicable conformity assessment standards guarantee the credibility and impartiality of the verification results assumed in the objectives set up for the EU ETV scheme. 


\section{AN ANALYSIS OF THE QUALITY ASSURANCE RESULTS OF THE EU ETV PILOT PROGRAMME}

In terms of capacity to perform verifications, the EU ETV scheme implemented as a pilot programme demonstrates sufficient development. There are currently 14 verification bodies from 7 countries providing services as entities of the EU ETV Pilot Programme. Accreditation of another one is pending. Except for Belgium, all countries which have been participating in the pilot from the beginning and Italy who joined later have established verification bodies. The largest number of these entities is observed in the United Kingdom (4) and in Poland (with 3 verification bodies established and one under accreditation procedure). Figure 1 presents the number of the verification bodies accredited in each of the countries taking part in the pilot.

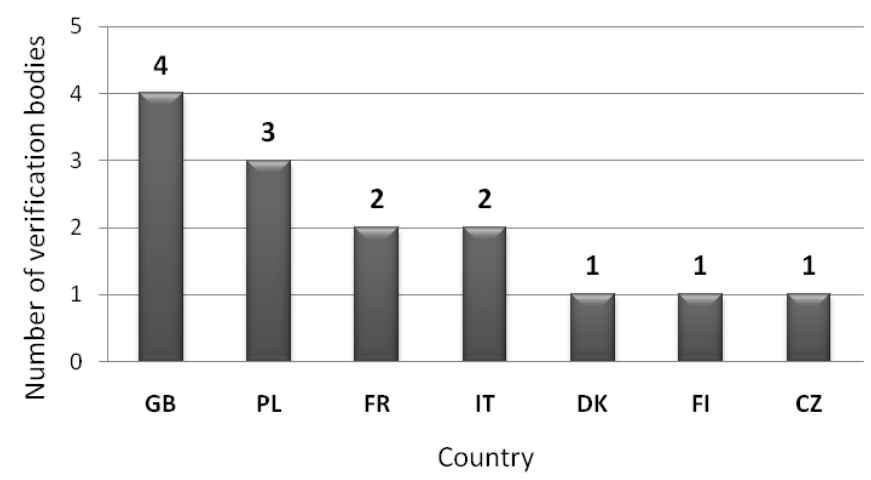

Fig. 1 The number of verification bodies which operate under the ETV Pilot Programme

Source: $h$ ttp://iet.jrc.ec.europa.eu/etv/.

Figure 2 presents the number of the accreditations of the verification bodies awarded in individual years of the pilot programme operation. The data shows that the dynamics of the accreditation process demonstrates a decreasing tendency. The highest number of accreditations was awarded in the years 2012-2014. It could be directly attributed to the fact that at that time the European Commission launched a call for proposals for potential verification bodies to offer financial support for them and in that way attempting to reduce the verification costs for the proposers. The condition to close a grant agreement with the Commission and get co-financing was a successfully completed accreditation for compliance to ISO/IEC 17020 for the application of GVP. It should be underlined the duration of the accreditation procedure could vary from country to country (typically lasting several months), so could the accreditation costs. Moreover, the duration does not include the preparatory phase during which all quality management documentation as well as operational procedures need to be drafted. In that context, the accreditation process can be described as a long-term activity and a costly investment process demonstrating business risk as the EU ETV Programme has been launched on an experimental basis with the decisions on the possible follow up upon assessment of the pilot by the end of 2017.

Figure 3 illustrates the distribution of verification bodies accredited for the three technology areas covered by the EU ETV Pilot Programme. The highest number of the verification bodies (13) has been accredited to perform verifications for technologies from the area "materials, waste and resources" followed by 11 bodies accredited to the energy technologies area. For the area "water treatment and monitoring" 10 verification bodies have been accredited.

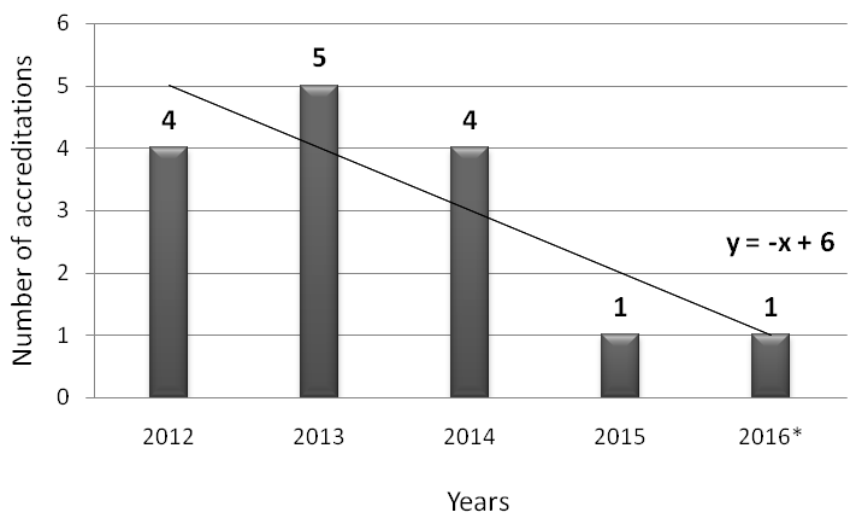

Fig. 2 The number of accreditations awarded in a given year for the Verification Bodies

Source: $h$ ttp://iet.jrc.ec.europa.eu/etv/.

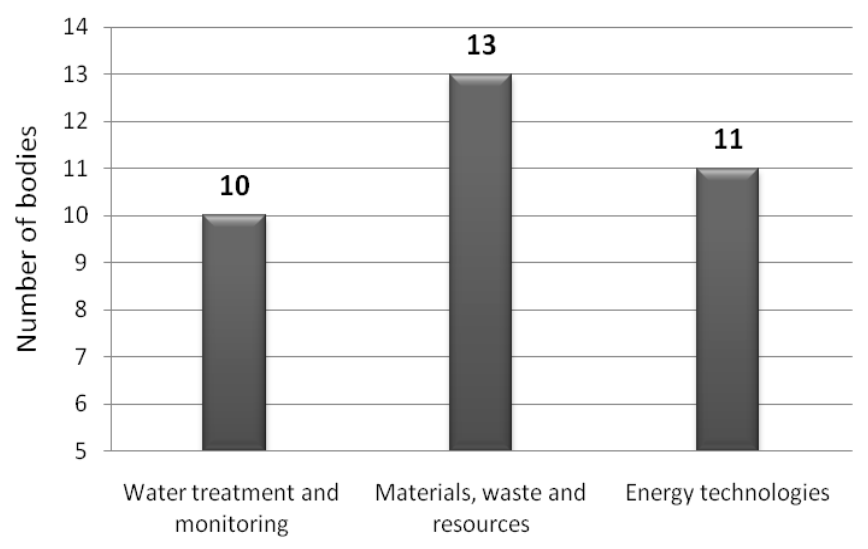

UE ETV Pilot Programme areas

Fig. 3 The number of accredited verification bodies with the division into accreditation areas under the UE ETV Pilot Programme Source: $h$ ttp://iet.jrc.ec.europa.eu/etv/.

As the data shows, majority of the verified bodies have been accredited for more than one technology area. Seven out of the 14 operating bodies is competent to perform verifications in all three areas of the pilot programme while 6 offer verifications in 2 areas. Only one body is accredited specifically to one area. Distribution of verification bodies with respect to the technology areas for which they have been accredited is presented in Figure 4.

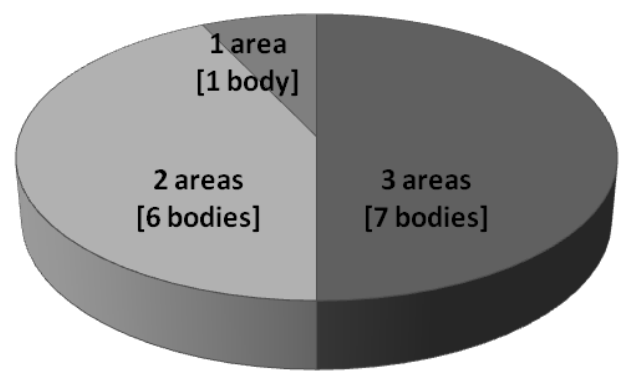

Fig. 4 The accreditation scope of verification bodies Source: $h$ ttp://iet.jrc.ec.europa.eu/etv/.

Verification bodies operate based on market conditions and are supposed to be financially sustainable. Without breaking the impartiality restrictions imposed by the ISO/ IEC 17020 standard for inspection bodies type A, the scope of services other than verification which they may offer is quite limited. According to the standard, a verification body 
and its personnel shall demonstrate that it is not linked to a party directly involved in design, manufacture, supply, installation, purchase, ownership, use or maintenance of the items inspected or similar competitive items. On the other hand, business success of these entities is clearly determined by the demand for the verification service. At the current stage of the EU ETV programme i.e. pilot phase, the market of ETV services can be assessed as risky, especially for the verification bodies. The ETV brand, although useful and undoubtedly supportive for promotion and market uptake of ecoinnovations is still not sufficiently recognised. Additionally the number of the verification bodies results in high competition at a limited interest of proposers. In that situation the decision of the verification bodies to cover all three technology areas in their accreditation seems fully justified as a broader scope of offered services allows to increase the chance for a market success.

Assuring the quality is also important in the context of the costs and duration of individual verifications. The European Commission assessed these costs on the level of $20000 €$, excluding the costs of potential testing. The costs of the verification body in the total verification costs must also include the costs associated with acquiring and maintaining the accreditation such as yearly audits, trainings of the personnel, etc. Moreover, the quality assurance requirements may generate extra costs of verification to be incurred by the proposers in the case when additional testing to produce quality test data is needed at the absence of appropriate evidence on the claimed performance of a technology. Although, as mentioned earlier, the EU ETV encourages the proposers to present test data generated prior to application for verification, practice demonstrates that in none of the verification procedures implemented so far, the existing data provided by the proposers met the quality requirements imposed by the ETV system. Moreover, in many cases additional controls and audits of the test bodies and the test systems that produce the test data has been required for compliance to ISO/IEC 17025 standard. Obviously these additional measures had a substantial impact on the costs and the duration of individual verifications. In certain circumstances it may become a serious entrance barrier, especially for SMEs.

The restrictive rules on quality assurance might also have an impact on the performance of the EU ETV Pilot Programme in terms of the number of procedures initiated, in progress and completed. Despite the fact that EU ETV was officially launched in December 2011, it gained full operational capacity at the moment when verification bodies covering all technology areas have become accredited. As it is showed on Fig. 2, majority of accreditations were awarded throughout of 2013. Taking into account the fact that by average a verification process lasts about a year since the initiation, the general output of the EU ETV is quite successful.

Until the end of March 2016, according to the data provided on the EU ETV Pilot Programme web site, 12 technologies were verified. In the case of 62 technologies contracts for verification were signed while as many as 175 quick scans were submitted. These figures allow for an optimistic outlook into the future of the verification market.

Figure 5 presents the number of technologies verified in individual years. The data shows a strong increasing tendency. Based only on the numbers presented in the figure a careful forecast can be made that the number of verifications will potentially double year after year.

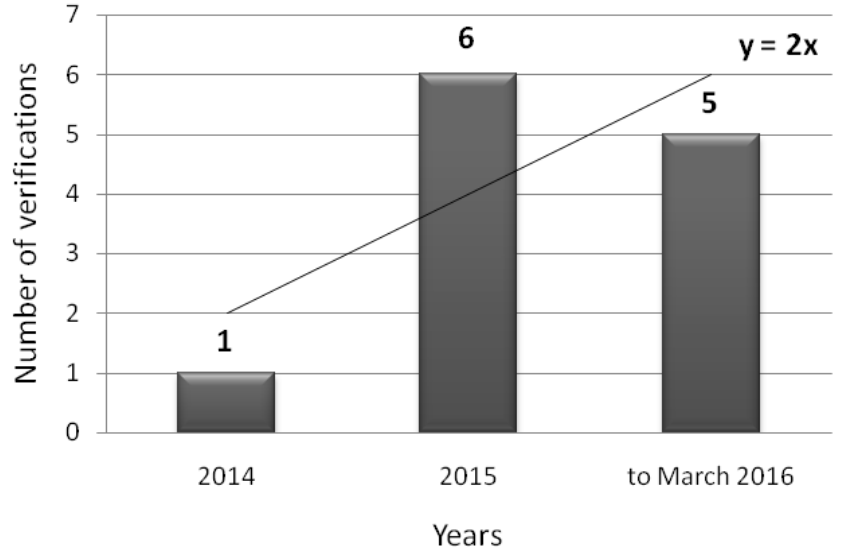

Fig. 5 The number of verification under the UE ETV Pilot Programme with the division into years Source: http://iet.jrc.ec.europa.eu/etv/.

The distribution of the numbers of technologies verified in individual countries is presented in Figure 6. Denmark is the leading country in terms of the highest number of verifications, followed by Italy. As many as 5 out of the 12 verifications under the EU ETV Pilot Programme were made in Denmark and 3 in Italy. In Poland 2 verifications were completed.

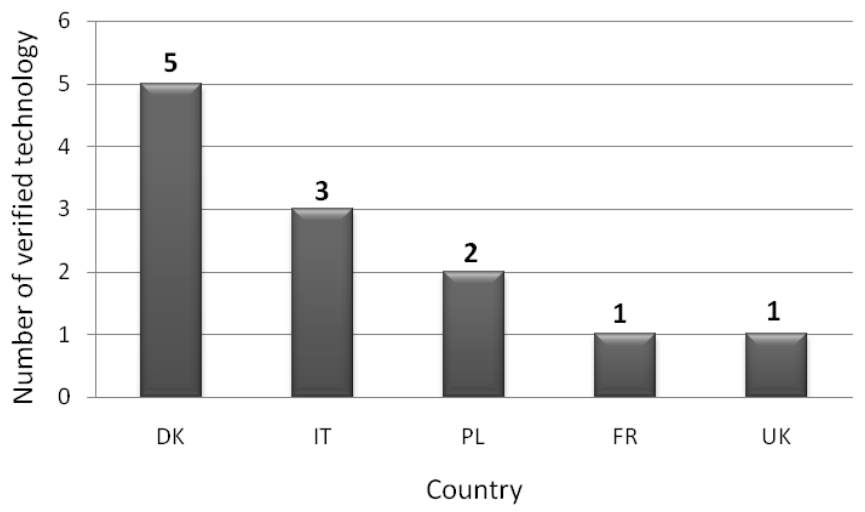

Fig. 6 The number of verified technologies with the division into UE country

Source: $h$ ttp://iet.jrc.ec.europa.eu/etv/.

Figure 7 illustrates the number of applications submitted in individual countries from the viewpoint of the origin of the proposers.

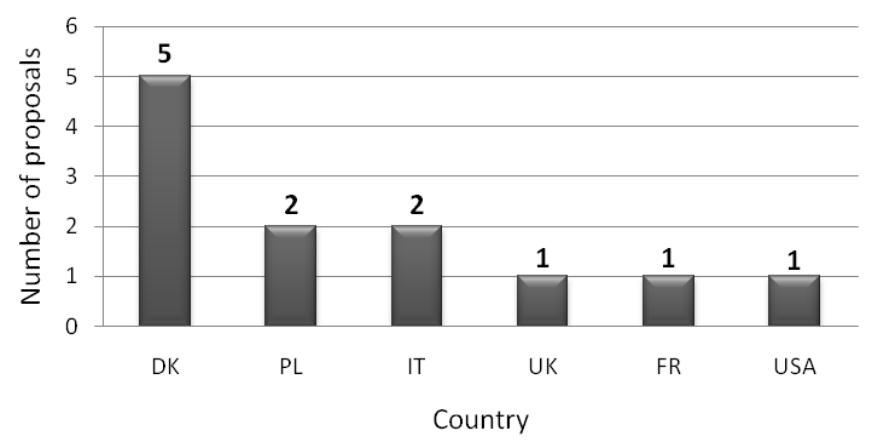

Fig. 7 The number of proposals with the division into the their origin

Source: $h$ ttp://iet.jrc.ec.europa.eu/etv/.

The data shows that despite the ability of the verification bodies to offer services to proposers originating from all over Europe and globally, the verifications performed so 
far are, in majority of cases, of local character i.e. the verification bodies offer services mainly to proposers from the same country in which they are established. Only in one case a manufacturer from the US applied for a verification under EU ETV scheme. Here the quality assurance issue may also have an impact. Compared to other systems, the EU ETV scheme represents the most restrictive requirements in terms of quality assurance covering both the verification bodies and the test data quality.

\section{CONCLUSION}

Quality assurance has been recognised as one of the priorities for the EU ETV scheme implemented under the Pilot Programme in response to the needs expressed by the users of the system. Vendors want to get a trustful confirmation on the performance of the technologies that they offer and the benefits provided by the technology. Technology purchasers and users want to get credible information on the technologies in order to choose solutions that fit best their specific needs and thus minimise the investment and technological risks associated with their implementation. The EU ETV scheme guarantees satisfaction of these demands. With its quality assurance framework based on commonly recognised ISO standards, not the case for other ETV schemes operating worldwide, and clear robust procedures described in the General Verification Protocol, the EU ETV scheme demonstrates the highest potential for the global recognition of the verification results.

This article was prepared within the statutory research titled "Methods and tools for improving products and services on the selected examples" work symbol 13/030/BK_17/0027 performed at Silesia University of Technology, Institute of Production Engineering.

\section{REFERENCES}

[1] S. Merkourakis, I. Calleja, L. Delgado, A. Oçafrain, S. Laurent "Environmental Technologies Verification Systems". [Online] Available: http://ftp.jrc.es/EURdoc/ eur22933en.pdf

[2] ENVIRONMENTAL TECHNOLOGY VERIFICATION, Consultations Analysis Report July 2008. [Online] Available: http://ec.europa.eu/environment/etv/pdf/ REPORT\%20consultations\%20on\%20ETV\%20final.pdf
[3] I. Ratman-Kłosińska, B. Michaliszyn, „Systemy wdrażania ekoinnowacji na świecie"; [w: Ekoinnowacje w praktyce funkcjonowania MŚP; red. L. Woźniak, J. Strojny, E. Wojnicka], PARP, Warszawa, 2010 r.

[4] Environmental Technology Verification. Test Report of Mobile Source Emission. Control Devices. [Online] Available:

[5] https://archive.epa.gov/nrmrl/archive-etv/web/ pdf/600etv07027.pdf

[6] J.A. Horwatich, S.R. Corsi, and R.T. Bannerman „Effectiveness of a pressurized stormwater filtration system in Green Bay, Wisconsin: A study for the environmental technology verification program of the US Environmental Protection Agency", US Department of the Interior, US Geological Survey, 2004. [Online] Available: http://pubs.usgs.gov/sir/2004/5222/pdf/ SIR_2004-5222.pdf

[7] C.W. Lee, D.G.Tabor, K.A. Cowen, „Environmental Technology Verification (ETV) test of dioxin emission monitors". [Online] Available: http:// link.springer.com/article/10.1007/s10163-007-0196-7

[8] T. Kelly, K. Hofacre, T. Derringer, D. Folsom, K. Riggs, „Testing of Safe Buildings Detection Technologies and Other Homeland Security Technologies in EPA's Environmental Technology Verification (ETV) Program". [Online] Available: http:// citeseerx.ist.psu.edu/viewdoc/download? doi=10.1.1.201.4132\&rep=rep1\&type=pdf

[9] M. Molenda, I. Ratman-Kłosińska, E. Sujova, Enviromental Technology Verification: The European Scheme as a New Quality in Validating The Performance of Ecoinnovation. [In]: 17th International Multidisciplinary Scientific GeoConference SGEM 2017. Conference Proceedings Volume 17. Ecology, economics, education and legislation, Issue 51. Ecology and Environmental Protection. Bulgaria, Albena, 2017.

[10] DETAILED ASSESSMENT OF THE MARKET POTENTIAL, AND DEMAND FOR, AN EU ETV SCHEME. [Online] Available: http://iet.jrc.ec.europa.eu/etv/referencedocuments

\footnotetext{
Eng. Michał Molenda PhD.

Silesian University of Technology

Faculty of Organization and Management

Institute of Production Engineering,

ul. Roosevelta 26, 41-800 Zabrze, POLAND

e-mail: michal.molenda@polsı.pl

MSc. Izabela Ratman-Kłosińska

Institute for Ecology of Industrial Areas

ul. Kossutha 6, 40-844 Katowice, POLAND

e-mail: i.ratman-klosinska@ietu.pl
} 